\title{
Dispersive magnetometry with a quantum limited SQUID parametric amplifier
}

\author{
M. Hatridge, ${ }^{1}$ R. Vijay, ${ }^{2}$ D. H. Slichter, ${ }^{2}$ John Clarke, ${ }^{1}$ and I. Siddiqi ${ }^{2}$ \\ ${ }^{1}$ Department of Physics, University of California, Berkeley, California 94720, USA and Materials Sciences Division, Lawrence Berkeley \\ National Laboratory, Berkeley, California 94720, USA \\ ${ }^{2}$ Quantum Nanoelectronics Laboratory, Department of Physics, University of California, Berkeley, California 94720, USA
}

(Received 11 November 2010; revised manuscript received 16 February 2011; published 4 April 2011)

\begin{abstract}
There is currently fundamental and technological interest in measuring and manipulating nanoscale magnets, particularly in the quantum coherent regime. To observe the dynamics of such systems one requires a magnetometer with not only exceptional sensitivity but also high gain, wide bandwidth, and low backaction. We demonstrate a dispersive magnetometer consisting of a two-junction superconducting quantum interference device (SQUID) in parallel with an integrated, lumped-element capacitor. Input flux signals are encoded as a phase modulation of the microwave drive tone applied to the magnetometer, resulting in a single quadrature voltage signal. For strong drive power, the nonlinearity of the resonator results in quantum limited, phase sensitive parametric amplification of this signal, which improves flux sensitivity at the expense of bandwidth. Depending on the drive parameters, the device performance ranges from an effective flux noise of $0.29 \mu \Phi_{0} \mathrm{~Hz}^{-1 / 2}$ and $20 \mathrm{MHz}$ of signal bandwidth to a noise of $0.14 \mu \Phi_{0} \mathrm{~Hz}^{-1 / 2}$ and a bandwidth of $0.6 \mathrm{MHz}$. These results are in excellent agreement with our theoretical model.
\end{abstract}

DOI: 10.1103/PhysRevB.83.134501

PACS number(s): 85.25.Dq, 85.25.Cp, 03.67.Ac

\section{INTRODUCTION}

The dc superconducting quantum interference device (SQUID) - a superconducting loop interrupted by two Josephson junctions-is an extremely sensitive detector of magnetic flux, and has been used in a wide variety of applications for almost half a century. ${ }^{1,2}$ Recent progress in nanoscale magnets ${ }^{3}$ has generated excitement about using magnetic molecules for both classical and quantum information storage and processing. ${ }^{4-12}$ In principle, a SQUID has sufficiently high intrinsic flux sensitivity and bandwidth to resolve the spin state of a single magnetic molecule. However, a conventional dc SQUID with resistively shunted Josephson tunnel junctions is operated with a current bias just above its critical current, and the continuous dissipation in the shunt resistors produces local heating and a backaction that can potentially induce relaxation and decoherence in a nanoscale magnet. This dissipation can be eliminated by using an unshunted SQUID and applying fast current pulses to measure its critical current. ${ }^{12,13}$ However, the flux sensitivity is significantly lower than in the resistively shunted case ${ }^{14}$ because the repetition rate-and hence the bandwidth - are limited by the time $(\sim 1 \mathrm{~ms}$ for aluminum tunnel junctions at millikelvin temperatures) required for the SQUID to cool to its equilibrium temperature after returning to the zero-voltage state. ${ }^{15}$ Any backaction associated with switching to the voltage state remains.

Alternatively, the SQUID can be operated in the superconducting regime where it functions as a flux dependent nonlinear inductor, and forms a nonlinear resonator when shunted with a capacitor [Fig. 1(a)]. In our device, we apply a fixed frequency microwave drive to this resonator and demodulate the reflected microwave signal. An input flux signal results in a variation of the resonance frequency and a corresponding phase modulation of the microwave drive tone. At specific bias points, in the presence of a sufficiently intense drive tone, parametric amplification occurs and the flux sensitivity is enhanced. Dispersive SQUID techniques have been studied in a variety of different microwave circuit configurations over the past 30 years. ${ }^{16-18}$ Recent work on the dispersive readout of superconducting qubits - single, pseudospin- $1 / 2$ systemsalso harnesses the nonlinearity of the Josephson junction to boost sensitivity, but typically these devices are operated in the bistable regime as digital detectors. ${ }^{19-21}$ In this paper, we demonstrate an analog magnetometer with megahertz bandwidth suitable for measuring transitions between states in multilevel spin systems ${ }^{22}$ and the macroscopic magnetization of spin ensembles. ${ }^{23}$ Depending on the operating conditions, the performance ranges from an effective flux noise of $0.14 \mu \Phi_{0} \mathrm{~Hz}^{-1 / 2}$ and $0.6 \mathrm{MHz}$ of signal bandwidth to a noise of $0.29 \mu \Phi_{0} \mathrm{~Hz}^{-1 / 2}$ and a bandwidth of $20 \mathrm{MHz}$. This performance results from the large gain, bandwidth, and nearly quantum limited noise temperature of the parametric amplifier which by itself is suitable as a general purpose amplifier. These results are in quantitative agreement with our theoretical model which, in particular, predicts that low flux noise and wide bandwidth are obtained for a low $Q$ (quality factor) resonator. Our theory allows us to optimize our device for specific applications, and provides insight into the fundamental and practical limitations of a single SQUID operated in the dispersive regime.

\section{THEORY}

We model our magnetometer as consisting of two stages: a transducer, which upconverts a low-frequency magnetic-flux signal to a microwave voltage signal, and a subsequent parametric gain stage [see Fig. 1(b)]. Using this picture, we derive an expression for the flux sensitivity based on the circuit parameters and the parametric gain. We first consider the dynamics of the Josephson oscillator. The supercurrent $I(t)$ flowing through a Josephson tunnel junction is related to the phase difference $\delta(t)$ across it by $I(t)=I_{0} \sin \delta(t)$, where $I_{0}$ is the critical current. For a SQUID with loop inductance $L \ll$ $\Phi_{0} / 2 I_{0}$, the critical current is $I_{c}(\Phi)=2 I_{0}\left|\cos \left(\frac{\pi \Phi}{\Phi_{0}}\right)\right|$, where $\Phi$ is the flux through the SQUID loop and $\Phi_{0} \equiv h / 2 e$ is the 

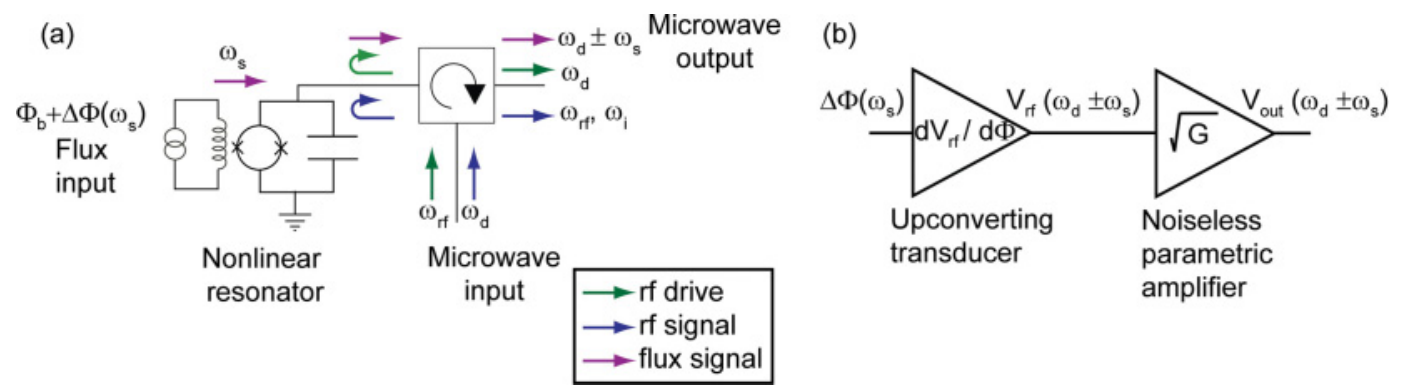

FIG. 1. (Color) (a) The magnetometer consists of the nonlinear inductance of an unshunted two-junction SQUID in parallel with an on-chip, lumped-element capacitor. An applied magnetic flux (purple arrows) modulates the resonant frequency, and is read out as a change in the phase of a microwave drive signal (green arrows) reflected from the device through a circulator. If the resonator is driven strongly, the upconverted flux signal (purple arrows) and any additional weak rf input signal (blue arrows) will be parametrically amplified. (b) The magnetometer can be characterized as a dual stage device, the first stage being an upconverting transducer of flux to microwave voltage and the second an rf parametric amplifier.

magnetic-flux quantum. Thus we treat the SQUID as a junction with a flux-dependent critical current. In our experiment the SQUID is shunted with a lumped-element capacitor, forming an electrical resonator with resonant frequency $\omega_{p 0}(\Phi) / 2 \pi=$ $\sqrt{2 \pi I_{c}(\Phi) /\left(\Phi_{0} C\right)} / 2 \pi$. The resonator is connected directly to a microwave transmission line of characteristic impedance $Z_{0}$ [Fig. 1(a)], resulting in a quality factor $Q=\omega_{p 0} Z_{0} C$.

The dynamics of this system are well described by the Duffing equation in which the sinusoidal current phase relationship of the junction is truncated after the first nonlinear term: $:^{24}$

$$
\frac{d^{2} \delta}{d t^{2}}+2 \Gamma \frac{d \delta}{d t}+\omega_{p 0}^{2}(\Phi)\left(\delta-\frac{\delta^{3}}{6}\right)=\frac{2 \pi}{\Phi_{0} C} I_{d} \cos \left(\omega_{d} t\right)
$$

Here, $\Gamma=\left(2 Z_{0} C\right)^{-1}$ and $I_{d}$ is the amplitude of the rf drive at frequency $\omega_{d} / 2 \pi$. Next we consider a flux $\Phi=\Phi_{b}+$ $\Delta \Phi \cos \left(\omega_{s} t\right)$ applied to the SQUID, where $\Phi_{b}$ is a static flux bias and $\Delta \Phi \ll \Phi_{0}$ is the amplitude of a weak flux signal at frequency $\omega_{s}$. We calculate the system response by assuming a solution of the form $\delta(t)=\delta_{0} \cos \left(\omega_{d} t-\theta\right)+\epsilon(t)$, where the first term is the steady-state solution for $\Delta \Phi=0$ and $\epsilon(t)$ is a small perturbation of the junction phase due to $\Delta \Phi$, and substituting it into Eq. (1). The resulting expression for the junction phase perturbation $\epsilon(t)$ is

$$
\begin{aligned}
\frac{d^{2} \epsilon}{d t^{2}} & +2 \Gamma \frac{d \epsilon}{d t}+\epsilon \omega_{p 0}^{2}\left(1-\frac{\delta_{0}^{2}}{4}\right)\left[1-\frac{\delta_{0}^{2}}{4-\delta_{0}^{2}} \cos \left(2 \omega_{d} t-2 \theta\right)\right] \\
& =\frac{V_{r f}}{Z_{0} C \Phi_{0}}\left[\cos \left(\omega_{d} t+\omega_{s} t-\theta\right)+\cos \left(\omega_{d} t-\omega_{s} t-\theta\right)\right] .
\end{aligned}
$$

We recognize the left-hand side of Eq. (2) as the equation for a parametrically driven harmonic oscillator. Details on the calculation of the gain and bandwidth of parametric amplification in this system are given in the Appendix. For appropriate bias conditions, the system can amplify ${ }^{25-27}$ any additional weak rf signal with frequency $\omega_{r f} / 2 \pi$ near $\omega_{d} / 2 \pi$. In this doubly degenerate mode of parametric amplification, ${ }^{27}$ a single sideband signal at frequency $\omega_{r f} / 2 \pi$ is amplified with a voltage gain $\sqrt{G}$, and an idler signal is also produced at frequency $\omega_{i} / 2 \pi=\left(2 \omega_{d}-\omega_{r f}\right) / 2 \pi$ with gain $\sqrt{G-1}$ and phase factor $e^{i \phi}$ [Fig. 2(a)]. In the high gain limit, the voltage signal-to-noise ratio (SNR) is degraded by a factor of at least $\sqrt{2}$, since the amplified signal is accompanied by incoherent noise from both the signal and idler frequencies. For an operating temperature $T \ll T_{Q}=\hbar \omega_{d} / 2 k_{B}$, this noise is set by the amplitude of quantum fluctuations at frequency $\omega_{d}$ and the amplifier is quantum limited with a noise temperature $T_{N}=T_{Q}$. Other Josephson-junction-based parametric amplifiers have been shown to operate with near quantum limited noise temperature. ${ }^{28,29}$ If such an amplifier is now presented with a double sideband signal, symmetric about the drive tone with coherent components at both the signal and idler frequencies, the output voltage is a coherent combination of these two signals. We can express this double sideband signal in terms of two orthogonal quadrature signals - one of which is amplified and the other de-amplified. This process is shown schematically for the amplified quadrature in Fig. 2(b). If the signal lies fully along the amplified quadrature $(\alpha=0)$, it is amplified without adding additional noise, a process known as phase-sensitive amplification. ${ }^{30}$

Examining the right-hand side of Eq. (2), we see that the flux signal at frequency $\omega_{s} / 2 \pi$ has been parametrically upconverted through interaction with the drive tone, resulting in a double sideband rf signal with components at frequencies $\left(\omega_{d} \pm \omega_{s}\right) / 2 \pi$ which can be expressed as a single quadrature signal with angle $\alpha=\theta$ relative to the drive tone. The voltage amplitude $V_{r f}$ of these two sidebands is linked to the input signal flux by the expression $V_{r f}=\Delta \Phi\left(d V_{r f} / d \Phi\right)$, where $d V_{r f} / d \Phi$ is the flux-to-voltage transduction gain, given by

$$
\begin{gathered}
\frac{d V_{r f}}{d \Phi}=\pi \frac{2 I_{0} Z_{0}}{4 \Phi_{0}} \sin \left(\frac{\pi \Phi_{b}}{\Phi_{0}}\right)\left(\delta_{0}-\frac{\delta_{0}^{3}}{8}\right), \\
\left(-\frac{\Phi_{0}}{2}<\Phi_{b}<\frac{\Phi_{0}}{2}\right) .
\end{gathered}
$$

In the limit of low drive current $I_{d} \ll I_{c}=8 I_{0} /\left(3^{3 / 4} Q^{3 / 2}\right)$ (see the Appendix for details of $G$ dependence on $I_{d}$ ), $\sqrt{G}=1$ and the paired rf signals serve to modulate the phase of the reflected microwave drive signal at frequency $\omega_{s} / 2 \pi$. In the high gain limit, the component of the transduced signal which lies along the amplified quadrature of the phasesensitive amplifier is noiselessly amplified with gain $2 \sqrt{G}$. The transduced signal, however, does not lie fully along the 
(a)

$$
\text { Parametric amplification }
$$
of a single sideband signal
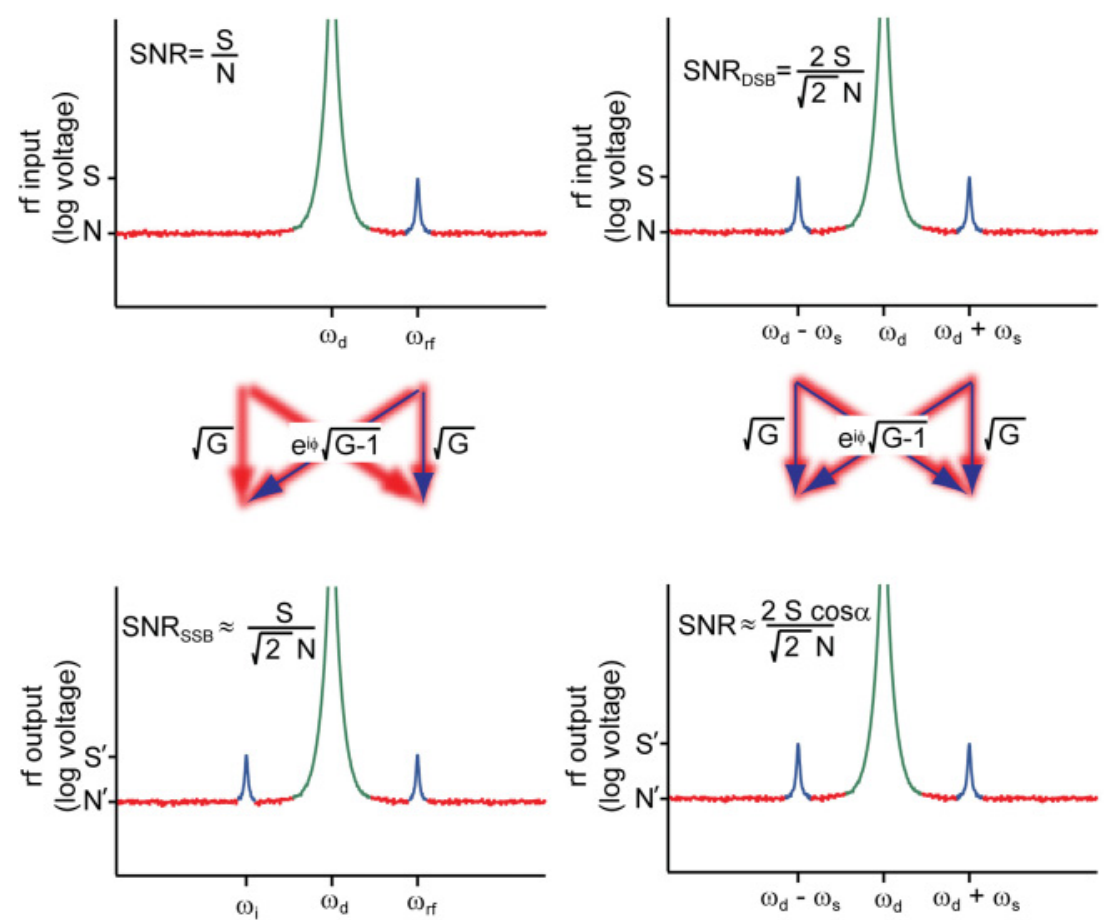

(b)

Parametric amplification of a double sideband signal

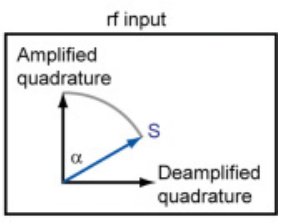

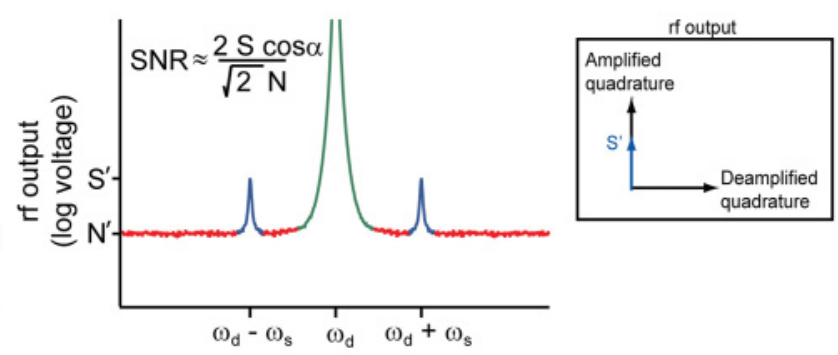

FIG. 2. (Color) Parametric amplification. The drive tone is in green, coherent signals are in blue, and incoherent noise is in red. (a) A single sideband signal at frequency $\omega_{r f} / 2 \pi$ is amplified with a voltage gain $\sqrt{G}$, and an idler signal is also produced at frequency $\omega_{i} / 2 \pi=\left(2 \omega_{d}-\omega_{r f}\right) / 2 \pi$ with gain $\sqrt{G-1}$ and relative phase $\phi$. In the high gain limit, the voltage signal-to-noise ratio (SNR) is degraded by a factor of at least $\sqrt{2}$, since the amplfied signal is accompanied by incoherent noise from both the signal and idler frequencies. (b) A coherent double sideband input signal symmetric about the drive tone results in output voltages which are a coherent combination of the signal and idler tones. This process can be decomposed as amplification of two orthogonal quadratures — one which is amplified and the other which is de-amplified. If the input signal lies fully along the amplified quadrature $(\alpha=0)$, it will be amplified without reduction of SNR.

amplified quadrature at high gain points so that the effective transduction coefficient is reduced. Consequently, in the high gain limit, the amplitude of the output signal is given by $\Delta \Phi \eta\left(d V_{r f} / d \Phi\right) \sqrt{G}$, where $\eta=2 \cos \theta$.

To characterize the flux sensitivity of the magnetometer, we first note that the output of our device is further amplified by a cryogenic high electron mobility transistor (HEMT) amplifier and a room temperature amplifier before being mixed down and digitized for further processing. The noise of this amplification chain is given by the system noise temperature $T_{\text {sys }}$, and includes cable losses between our device and the HEMT. We now refer the noise of the output rf voltage signal to an effective input flux noise via the transduction coefficient $\eta\left(d V_{r f} / d \Phi\right)$. This effective flux noise has a single-sided spectral density,

$$
\begin{aligned}
S_{\Phi \mathrm{eff}}^{1 / 2}(f) & =\frac{\sqrt{2\left(k_{B} T_{\mathrm{sys}}+\hbar \omega_{d} / 2\right) Z_{0}}}{\eta \frac{d V_{r f}}{d \Phi} \Phi_{0}} \\
& =\frac{4}{\pi} \frac{\sqrt{2\left(k_{B} T_{\mathrm{sys}}+\hbar \omega_{d} / 2\right) Z_{0}}}{2 I_{0} Z_{0} \eta \sin \left(\frac{\pi \Phi_{b}}{\Phi_{0}}\right)\left(\delta_{0}-\frac{\delta_{0}^{3}}{8}\right)} \quad \frac{\Phi_{0}}{\sqrt{\mathrm{Hz}}} .
\end{aligned}
$$

Note that in the above expression $T_{\text {sys }}$ refers to the added noise of the amplification chain and the total noise includes a contribution from zero-point fluctuations given by $\hbar \omega_{d} / 2$.
For weak drive amplitudes, where the resonator response is linear and $G=1, T_{\text {sys }}$ is dominated by $T_{\text {HEMT }}$, the noise temperature of the HEMT amplifier, and is typically much larger than the quantum limit. Furthermore, we can express the junction oscillation amplitude in terms of the voltage drive as $\delta_{0}-\delta_{0}^{3} / 8 \approx \delta_{0} \approx V_{d} Q / Z_{0} 2 I_{0}$. In the absence of parametric amplification, there is no preferred quadrature for amplification and we set $\eta=1$ to yield

$$
S_{\Phi \mathrm{eff}}^{1 / 2}(f) \approx \frac{4}{\pi} \frac{\sqrt{2 k_{B} T_{\mathrm{HEMT}} Z_{0}}}{\sin \left(\frac{\pi \Phi_{b}}{\Phi_{0}}\right) V_{d} Q} \quad \frac{\Phi_{0}}{\sqrt{\mathrm{Hz}}} \quad \text { for } \quad G=1,
$$

which varies inversely with resonator $Q$ and drive voltage $V_{d}$. Thus in this regime higher sensitivity can be achieved by increasing the $Q$ of the resonator and the drive amplitude. In practice, however, the maximum drive voltage is limited by the onset of nonlinearity inherent in any junction-based resonator. $^{31}$

For strong drive amplitudes, where the resonator response is nonlinear and $G \gg 1$ such that $T_{\text {HEMT }} / G \ll \hbar \omega_{d} / 2$, the noise temperature of the system is determined by the amplitude of quantum fluctuations at the drive frequency, and $T_{\text {sys }} \approx$ $\hbar \omega_{d} / 2 k_{B}$. Further, as $G \rightarrow \infty, \delta_{0}$ approaches a critical value ${ }^{31}$ of $\delta_{c}=4 / 3^{1 / 4} \sqrt{Q}$. For $Q \gtrsim 10, \delta_{c} \leqslant 1$ and we can make the approximation $\delta_{0}-\delta_{0}^{3} / 8 \approx \delta_{c}$. Similarly, at this operating 
point, the angle $\theta$ also approaches a critical value of $60^{\circ}$, so that we can approximate $\eta \approx 1$. Since the maximum value of $\eta$ is 2 , only half of the transduced signal is noiselessly amplified resulting in an effective flux noise two times higher than what could be achieved in the ideal case with $\theta=0$. A similar analysis, which treats the system as a degenerate parametric amplifier with a detuned pump and takes into account the nonorthogonality of the amplified and de-amplified quadratures, gives the same result in this limiting case. $^{32}$ The effective flux noise computes to

$$
\begin{array}{r}
S_{\Phi \text { eff }}^{1 / 2}(f) \approx \frac{(2 \sqrt{3})^{1 / 2}}{\pi} \frac{\sqrt{\hbar \omega_{d}}}{2 I_{0} \sin \left(\pi \Phi_{b} / \Phi_{0}\right)} \sqrt{\frac{Q}{Z_{0}}} \frac{\Phi_{0}}{\sqrt{\mathrm{Hz}}} \\
\text { for } \quad G \gg 1 .
\end{array}
$$

This expression has the remarkable feature that the effective flux noise varies directly with the resonator $Q$, so that a resonator with lower $Q$ has improved flux sensitivity. This results from the requirements of parametric amplification, in particular that high parametric gain occurs at a critical phase oscillation $\delta_{c} \propto Q^{-1 / 2}$, which in turn limits the maximum achievable transduction coefficient. Additionally, lowering the resonator $Q$ leads to parametric amplification with increased bandwidth for a given parametric gain, and so is doubly desirable. This advantage breaks down at very low $Q$, where the resonator oscillations will become chaotic before reaching the critical phase oscillation point, ${ }^{31}$ limiting the achievable parametric gain and associated flux sensitivity. In both the linear and nonlinear regime, it is advantageous to increase the SQUID critical current and operate at a flux bias near $\Phi_{0} / 2$. However, for sufficiently large critical current such that $\Phi_{0} / I_{0} \sim L$, where $L$ is the inductance of the SQUID loop, the simple expression for $I_{c}(\Phi)$ is no longer valid and one has to use numerical simulations to determine the change in SQUID inductance as a function of applied flux, which is often a multivalued function. Furthermore, unlike the critical current modulation of a dc SQUID, the inductance modulation with flux of a dispersive SQUID is not limited by the loop inductance provided one remains in one of the branches of the multivalued function.

\section{EXPERIMENT}

Our lumped element resonator is shown in a false color scanning electron microscope image in Fig. 3(a). The device consists of three layers: a 250-nm-thick $\mathrm{Nb}$ underlayer, a 180-nm-thick $\mathrm{SiN}_{x}$ insulating layer, and an aluminum upper layer. The capacitor was fabricated in a split geometry, with both electrodes on the top Al layer coupled through the Nb underlayer. The split geometry simplifies fabrication by avoiding the use of vias. The SQUID was fabricated with double-angle evaporated $\mathrm{Al}-\mathrm{AlO}_{x}-\mathrm{Al}$ junctions, with $2 I_{0}=4.3 \mu \mathrm{A}$. A shortcircuit terminated coplanar waveguide transmission line was also fabricated on-chip to apply oscillating flux signals to the SQUID loop. Static flux was provided by a superconducting wire-wound coil. The device was cooled in superconducting and Cryoperm shields, and its performance measured at $30 \mathrm{mK}$ in a cryogen-free dilution refrigerator. All static and rf lines were heavily attenuated and filtered to minimize external noise.

To determine the dependence of the resonant frequency on applied flux, we used a vector network analyzer to measure the phase of a weak microwave tone reflected from the resonator as a function of frequency and flux. The results are shown in Fig. 3(b). As the flux through the SQUID washer was varied, the resonant frequency varied from a maximum of $7.2 \mathrm{GHz}$ to a minimum of $4 \mathrm{GHz}$, set by the low-frequency cutoff of the circulators used in the measurement chain. The horizontal bands apparent in the plot are due to the finite directivity of the circulator used to separate the incoming and outgoing microwave signals from the resonator. The sample was flux biased at $\Phi_{b}=0.3 \Phi_{0}$, where the flux sensitivity was high and the frequency band was clear of ripples which could obscure the response to $\mathrm{rf}$ and magnetic signals.

We next examined the performance of the parametric amplification stage. To characterize the gain, a strong rf drive tone at frequency $\omega_{d} / 2 \pi$ was applied to the resonator simultaneously with a weak rf signal at frequency $\omega_{r f} / 2 \pi$ [Fig. 3(a)]. The reflected rf signal was further amplified, demodulated by a double sideband mixer with local oscillator frequency $\omega_{L O} / 2 \pi=\omega_{r f} / 2 \pi+110 \mathrm{~Hz}$, and digitized. The gain was determined by calculating the ratio of the reflected microwave signal with the drive tone turned on versus a calibration sweep with the drive tone turned off; in the latter
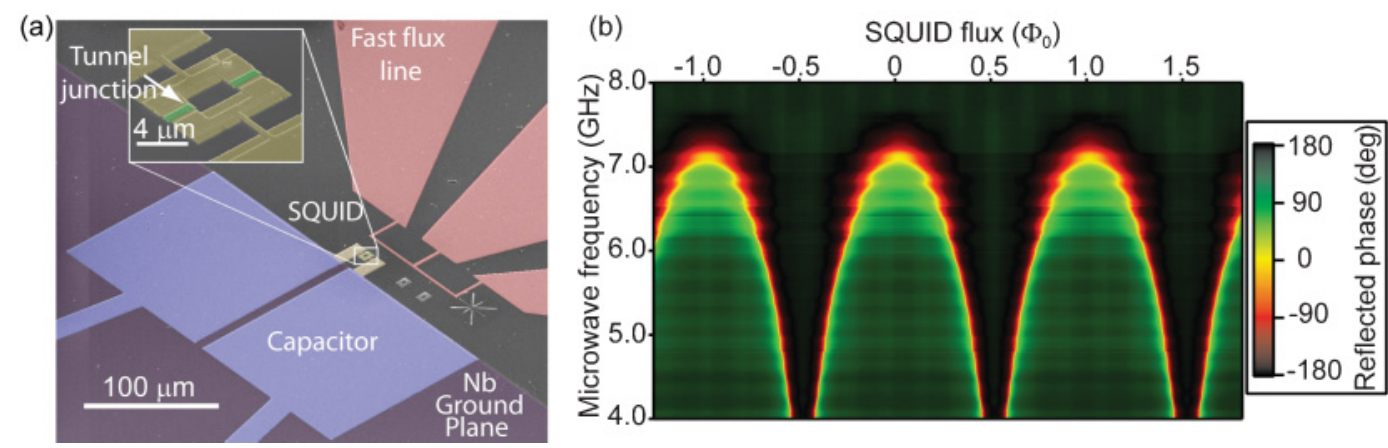

FIG. 3. (Color) (a) False color scanning electron microscope image of the dispersive magnetometer. The SQUID is shown magnified in the inset, with the $\mathrm{Al}-\mathrm{AlO}_{x}-\mathrm{Al}$ tunnel junctions marked in green. The capacitor is formed in a split geometry, with two top layer electrodes connected through a common niobium plane. A high bandwidth flux line is formed by a short-circuit terminated coplanar waveguide transmission line, shown in the upper right of the figure. (b) The phase of the reflected microwave drive is plotted versus applied SQUID flux and drive frequency. Each vertical slice is a resonance curve, with the color yellow (zero phase) representing the resonant frequency. 
(a)

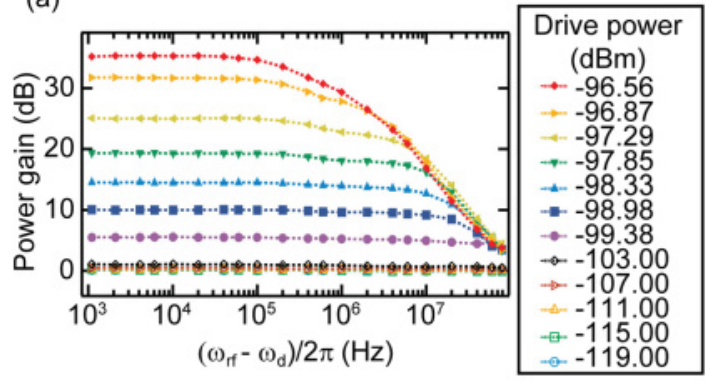

(c)

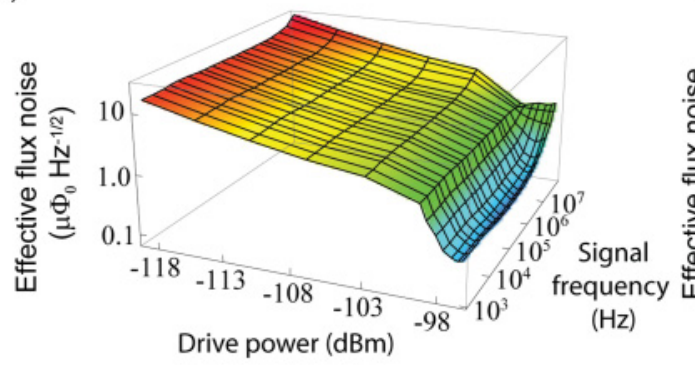

(b)

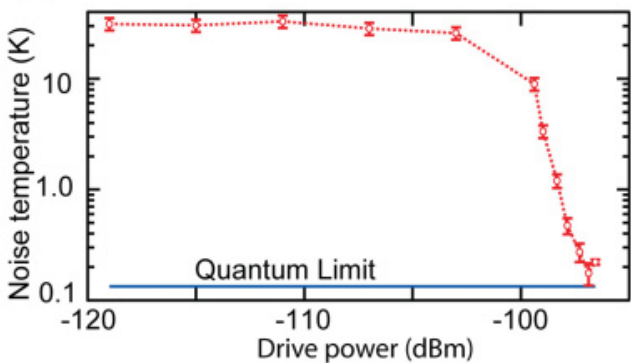

(d)

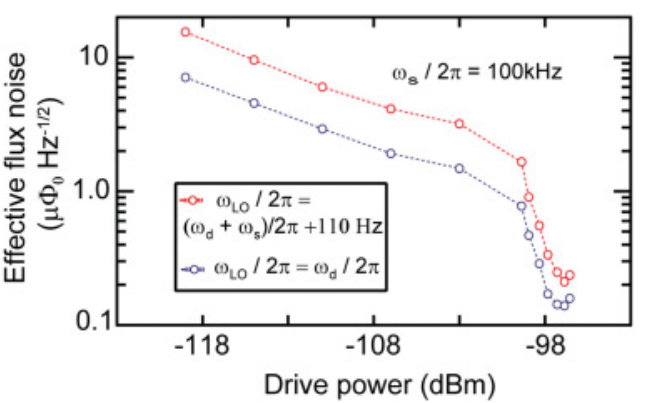

FIG. 4. (Color) (a) The parametric gain of rf signals $\left(\omega_{r f} / 2 \pi\right)$ applied in combination with an rf drive tone $\left(\omega_{d} / 2 \pi\right)$ versus the offset frequency $\left(\omega_{r f}-\omega_{d}\right) / 2 \pi$. (b) System noise temperature versus drive power. As the parametric gain increases with drive power, the system noise temperature drops. The standard quantum limit $T_{N}=\hbar \omega_{d} / 2 k_{B}$ at $\omega_{d} / 2 \pi=5.56 \mathrm{GHz}$ is shown as a blue horizontal line. (c) Effective flux noise versus microwave drive power and flux signal frequency $\omega_{s} / 2 \pi$. As the system noise temperature decreases with drive power, the effective flux noise is reduced. (d) Effective flux noise versus drive power for two demodulation schemes. Data from part (c) at $\omega_{s} / 2 \pi=100 \mathrm{kHz}$ and demodulated with $\omega_{L O} / 2 \pi=\left(\omega_{d}+\omega_{s}\right) / 2 \pi+110 \mathrm{~Hz}$, shown in red, are compared with those demodulated with $\omega_{L O}=\omega_{d}$, shown in blue. At each bias point, the phase of the LO signal was varied to achieve maximum sensitivity. The latter demodulation scheme shows the expected noise improvement factor of 2 for $\sqrt{G}=1$, and $\sqrt{2}$ for $\sqrt{G} \gg 1$.

mode the signal tone is reflected from the resonator with unity gain. The gain is plotted in Fig. 4(a) as a function of the frequency offset $\left(\omega_{r f}-\omega_{d}\right) / 2 \pi$ for different drive powers. The advantage of low resonator $Q$ is evident in these data where a gain of $15 \mathrm{~dB}$ is demonstrated with a $3-\mathrm{dB}$ bandwidth of $20 \mathrm{MHz}$. We note that the observed gain and bandwidth deviate slightly from the analytical solution of the simple Duffing oscillator prediction for a given drive amplitude. This discrepancy is resolved using numerical simulations which indicate gain suppression due to noise rounding in nonlinear resonators with very low $Q$.

In separate measurements using a hot/cold load, we found the system noise temperature at the plane of the resonator to be between 29 and $37.5 \mathrm{~K}$, with the scatter arising from the uncertainty in the attenuation of the hot load line. By measuring the SNR improvement of rf signals measured at finite gain versus $G=0 \mathrm{~dB}$, we calculate the system noise temperature as a function of drive power [shown in Fig. 4(b)]. The lowest system noise temperature was measured with $G=32 \mathrm{~dB}$ at $\omega_{d} / 2 \pi=5.56 \mathrm{GHz}$, with $T_{\text {sys }}$ between 0.14 and $0.21 \mathrm{~K}$, corresponding to a nearly quantum limited added noise of 0.50 to 0.78 photons. At the highest gain point, the noise temperature was degraded due to instabilities associated with operation near the critical point. ${ }^{33}$

We subsequently investigated the flux response of the system by applying a flux tone at frequency $\omega_{s} / 2 \pi$, chosen so that the upper sideband of the upconverted rf output at frequency $\left(\omega_{d}+\omega_{s}\right) / 2 \pi$ was offset by $10 \mathrm{~Hz}$ from an additional applied rf signal at frequency $\omega_{r f} / 2 \pi$. This allows simultaneous measurements of the parametric gain and flux sensitivity. The output signals were again amplified, demodulated with a double sideband mixer with local oscillator frequency $\omega_{L O} / 2 \pi=\omega_{r f} / 2 \pi+110 \mathrm{~Hz}$, and digitized. The SNR of the flux response at each bias point was calculated by comparing the height of the transduced output signal to the average of the white noise in a bandwidth of $200 \mathrm{~Hz}$. This SNR was converted into an effective flux noise using the known amplitude of the flux signal applied to the magnetometer. In these measurements, the primary source of uncertainty in the effective flux noise is the calibration of the flux signal, which we estimate to be a few percent. In Fig. 4(c), we plot the effective flux noise as a function of drive amplitude and flux signal frequency. In the linear regime, the magnetometer bandwidth is limited by the bandwidth of our flux excitation line and is demonstrated to be at least $80 \mathrm{MHz}$. Using our value of $Q \approx 26$, we expect the bandwidth to be greater than $100 \mathrm{MHz}$. By biasing the resonator in the nonlinear regime, we trade bandwidth for parametric gain and reduced noise. By operating at a parametric gain of $32 \mathrm{~dB}$, we achieved a minimum effective flux noise of $0.21 \mu \Phi_{0} \mathrm{~Hz}^{-1 / 2}$ at $\omega_{s} / 2 \pi=$ $100 \mathrm{kHz}$. However, this is not the lowest attainable noise as the demodulated noise in these measurements with $\omega_{L O} \neq \omega_{d}$ 
is the sum of incoherent noise sidebands above and below the LO frequency, thus degrading the effective flux noise.

To determine the optimum device performance, we performed a second set of measurements with $\omega_{L O}=\omega_{d}$. With this demodulation technique we made use of the single quadrature nature of the transduced flux signal. The effective flux noise as function of drive power measured at $\omega_{s} / 2 \pi=100 \mathrm{kHz}$ for both demodulation techniques is shown in Fig. 4(c) and clearly demonstrates the advantage of demodulating with $\omega_{L O}=\omega_{d}$. In the linear regime at low powers, the latter demodulation scheme improves the effective flux noise by a factor of 2 since both sidebands containing information are used. At high drive powers the improvement is only $\sqrt{2}$, since both sidebands contain identical signal and noise due to parametric amplification. The minimum effective flux noise of $0.14 \mu \Phi_{0} \mathrm{~Hz}^{-1 / 2}$ was achieved with $G=32 \mathrm{~dB}$, with a flux signal bandwidth of $600 \mathrm{kHz}$ set by the parametric amplifier bandwidth. If we substitute our system parameters into Eq. (6), we predict a minimum effective flux noise of $0.14 \pm 0.007 \mu \Phi_{0} \mathrm{~Hz}^{-1 / 2}$, in very good quantitative agreement with our measured results. The uncertainty in this calculation is the estimated uncertainty in our knowledge of the circuit parameters. By reducing the parametric gain to $15 \mathrm{~dB}$, we achieved an effective flux noise of $0.29 \mu \Phi_{0} \mathrm{~Hz}^{-1 / 2}$ while significantly increasing the flux signal bandwidth to $20 \mathrm{MHz}$. As a final note, at frequencies below $100 \mathrm{~Hz}$ (not shown) we observe an effective flux noise with a nearly $1 / f$ power spectrum and a value of a few $\mu \Phi_{0} \mathrm{~Hz}^{-1 / 2}$ at $1 \mathrm{~Hz}$, consistent with that typically measured for SQUIDs at low temperatures. $^{34}$

\section{CONCLUSION}

We have demonstrated a dispersive magnetometer based on a lumped-element, nonlinear resonator involving a twojunction SQUID. The bandwidth and sensitivity of the device can be dynamically altered to suit the needs of specific measurements by changing the microwave drive. We have achieved an effective flux noise of $0.14 \mu \Phi_{0} \mathrm{~Hz}^{-1 / 2}$ with a bandwidth of $600 \mathrm{kHz}$. The bandwidth can be increased to $20 \mathrm{MHz}$ with only a factor of 2 increase in flux noise. We expect that our magnetometer will exhibit low backaction, since the SQUID never switches to the voltage state, making it attractive for quantum state measurement. There are several avenues for further improvement. The magnetometer could be realized as two physically separate devices: a transducer and a gain stage. This would allow for independent optimization of the transduction coefficient and the performance of the parametric amplifier. By increasing the $Q$ of the transduction stage to match the bandwidth of the parametric amplifier, and rotating the transduced signal fully into the amplified quadrature, reductions by a factor of about 10 in the effective flux noise should be possible. A lower effective flux noise could also be achieved by optimizing the transduction coefficient using junctions with higher critical currents, and by operating at a flux bias closer to $\Phi_{0} / 2$. An increased bandwidth could be obtained by reducing the noise temperature of the microwave postamplifier, thus reducing the parametric gain necessary for quantum noise limited operation. Moreover, the quantum noise limited amplification we have observed suggests that this device can be used as a general purpose first-stage rf amplifier for a variety of applications.

An attractive possibility is to replace the tunnel junctions in the SQUID with nanobridges, provided they have sufficient nonlinearity to exhibit efficient flux transduction and high parametric gain. ${ }^{35,36}$ Furthermore, nanobridges are resilient to applied longitudinal magnetic fields. Nanobridges also have the important advantage of overcoming the inherent difficulty of coupling a nanoscale spin system, such as a single molecule magnet (SMM), to a SQUID loop with a diameter of the order of a micrometer. By placing the SMM directly across the bridge with nanometer dimensions, one can achieve high coupling efficiency. ${ }^{12}$ Such experiments would enable one to investigate the all-important issue of backaction from the SQUID on the SMM.

\section{ACKNOWLEDGMENTS}

The authors thank O. Naaman for helpful discussions. This work was supported by the Director, Office of Science, Office of Basic Energy Sciences, Materials Sciences and Engineering Division, of the US Department of Energy under Contract No. DE-AC02-05CH11231(M.H., J.C.). Financial support was also provided by the Office of Naval Research under Grant No. N00014-07-1-0774 (I.S.) and AFOSR Grant No. FA9550-08-1-0104 (R.V., I.S.). D.H.S. acknowledges support from a Hertz Foundation Fellowship endowed by Big George Ventures.

\section{APPENDIX: DERIVATION OF PARAMETRIC AMPLIFIER GAIN}

In this Appendix, we derive the expressions for parametric gain and consider the limiting cases. We start with a modified version of Eq. (2), replacing the right-hand side by a signal at frequency $\omega_{d}+\omega_{s}$. This represents the application of a weak signal tone to the nonlinear oscillator in addition to the strong pump tone at $\omega_{d}$,

$$
\begin{aligned}
& \frac{d^{2} \epsilon}{d t^{2}}+2 \Gamma \frac{d \epsilon}{d t}+\epsilon \omega_{p 0}^{2}\left(1-\frac{\delta_{0}^{2}}{4}\right)\left[1-\frac{\delta_{0}^{2}}{4-\delta_{0}^{2}} \cos \left(2 \omega_{d} t-2 \theta\right)\right] \\
& =\frac{1}{Z_{0} C \Phi_{0}} V_{s}(t),
\end{aligned}
$$

where $V_{s}(t)=V_{s} \cos \left(\omega_{d} t+\omega_{s} t\right)$ is the signal tone which will be amplified. We next decompose $\delta_{0}$ into components that are parallel and perpendicular to the phase of the pump tone, yielding $\delta_{0}^{2}=\delta_{c \perp}^{2}+\delta_{c \|}^{2}$, where $\tan \theta=\delta_{c \perp} / \delta_{c \|}$. By substituting this expression into Eq. (1) and making the rotating wave approximation (RWA), we arrive at

$$
-\delta_{c \|}+\delta_{c \perp}\left[\Omega-\frac{Q}{8}\left(\delta_{c \perp}^{2}+\delta_{c \|}^{2}\right)\right]=0
$$

and

$$
\delta_{c \perp}+\delta_{c \|}\left[\Omega-\frac{Q}{8}\left(\delta_{c \perp}^{2}+\delta_{c \|}^{2}\right)\right]=Q I_{d} / I_{0},
$$

where $\Omega=2 Q\left(1-\omega_{d} / \omega_{p 0}\right)$ is the dimensionless detuning of the pump tone from the resonant frequency $\omega_{p 0}$. These 
expressions allow us to solve for $\delta_{0}$ as a function of drive parameters.

As the parametric amplifier operates in a reflection mode, it is next useful to represent the signal tone in terms of incident $\left(V_{\text {in }}\right)$ and reflected $\left(V_{\text {out }}\right)$ amplitudes. Using ideas from inputoutput theory, ${ }^{37}$ we write

$$
V_{s}(t)=2 V_{\text {in }}(t)
$$

and

$$
V_{\text {out }}(t)=V_{J}(t)-V_{\text {in }}(t) .
$$

Here $V_{J}(t)=\left(\Phi_{0} / 2 \pi\right) d \epsilon / d t$ is the voltage across the Josephson junction. For harmonic signals, the above system of equations can be solved analytically in the frequency domain. ${ }^{27}$ The signal power gain $\left(G_{s}\right)$ is defined to be the ratio of output power to input power at the signal frequency $\omega_{d}+\omega_{s}$ and is given by

$$
G_{s}(f)=1+\frac{4 \rho^{2}}{\left[(\Omega-2 \rho)^{2}-\rho^{2}+1\right]^{2}-2 f^{2}\left[(\Omega-2 \rho)^{2}-\rho \epsilon^{2}-1\right]+f^{4}},
$$

where $\rho=Q \delta_{0}^{2} / 8$ and $f=2 Q\left(1-\omega_{s} / \omega_{p 0}\right)$ is the dimensionless signal frequency. For small offset frequencies $\omega_{s}$, the gain is flat versus offset frequency, and this gain $(G)$ can be expressed versus bias conditions $(\Omega, \rho)$ by setting $f=0$, yielding

$$
G=1+\frac{4 \rho^{2}}{\left[(\Omega-2 \rho)^{2}-\rho^{2}+1\right]^{2}} .
$$

Note that the above expression depends only on the dimensionless pump frequency $(\Omega)$ and oscillation amplitude $(\rho)$ at the pump frequency.

We next analyze the behavior of $G$ in the limiting cases. For no pump drive, i.e., $\rho=0$, we obtain $G=1$ as expected. For $\Omega<\sqrt{3}$, the maximum gain for a given pump frequency is achieved when

$$
\rho=\rho_{\max }=\sqrt{\frac{1+\Omega^{2}}{3}}
$$

and

$$
I_{d}^{2}=\frac{16 I_{0}^{2}}{3 \sqrt{3} Q^{3}}\left(1+\Omega^{2}\right)\left(2 \sqrt{1+\Omega^{2}}-\sqrt{3} \Omega\right),
$$

where $I_{d}$ is the pump drive amplitude and the maximum gain is given by

$$
G_{\max }=1+\frac{1}{3+7 \Omega^{2}-4 \sqrt{3} \Omega \sqrt{1+\Omega^{2}}} .
$$

At the critical point $(\Omega=\sqrt{3})$ the gain $\left(G_{\max }\right)$ diverges. In practice, higher-order corrections ignored in this calculation will ensure that the gain remains finite. We always operate with $\Omega<\sqrt{3}$ to avoid instabilities in the system when it is operated very near the critical point.
${ }^{1}$ The SQUID Handbook, edited by J. Clarke and A. I. Braginski (Wiley, Weinheim, 2004), Vol. 1.

${ }^{2}$ The SQUID Handbook (Ref. 1), Vol. 2.

${ }^{3}$ S. D. Bader, Rev. Mod. Phys. 78, 1 (2006).

${ }^{4}$ M. Leuenberger and D. Loss, Nature (London) 410, 789 (2001).

${ }^{5}$ J. Tejada, E. Chudnovsky, E. del Barco, J. Hernandez, and T. Spiller, Nanotechnology 12, 181 (2001).

${ }^{6}$ J. Gallop, P. Josephs-Franks, J. Davis, L. Hao, and J. Macfarlane, Physica C 368, 109 (2002).

${ }^{7}$ S. Lam and D. Tilbrook, Appl. Phys. Lett. 82, 1078 (2003).

${ }^{8}$ D. Gatteschi, R. Sessoli, and J. Villain, Molecular Nanomagnets (Oxford University Press, New York, 2006).

${ }^{9}$ J.-P. Cleuziou, W. Wernsdorfer, V. Bouchiat, T. Ondarcuhu, and M. Monthioux, Nat. Nano. 1, 53 (2006).

${ }^{10}$ A. Troeman, H. Derking, B. Borger, J. Pleikies, D. Veldhuis, and H. Hilgenkamp, Nano Lett. 7, 2152 (2007).

${ }^{11}$ C. Granata, E. Esposito, A. Vettoliere, L. Petti, and M. Russo, Nanotechnology 19, 275501 (2008).

${ }^{12}$ W. Wernsdorfer, Supercond. Sci. Technol. 22, 064013 (2009).

${ }^{13}$ C. van der Wal, A. ter Haar, F. Wilhelm, R. Schouten, C. Harmans, T. Orlando, S. Lloyd, and J. Mooij, Science 290, 773 (2000).
${ }^{14}$ D. J. Van Harlingen, R. H. Koch, and J. Clarke, Appl. Phys. Lett. 41, 197 (1982)

${ }^{15}$ K. Segall, C. Wilson, L. Li, L. Frunzio, S. Friedrich, M. C. Gaidis, and D. E. Prober, Phys. Rev. B 70, 214520 (2004).

${ }^{16} \mathrm{~K}$. Likharev, Dynamics of Josephson Junctions and Circuits (Gordon and Breach, New York, 1985).

${ }^{17}$ L. Kuzmin, K. Likharev, V. Migulin, E. Polunin, and N. Simonov, in SQUID'85, Superconducting Quantum Interference Devices and Their Applications, edited by H. Hahlbohm and H. Lübbig (de Gruyter, Berlin, 1985), pp. 1029-1034.

${ }^{18}$ J. A. B. Mates, G. C. Hilton, K. D. Irwin, L. R. Vale, and K. W. Lehnert, Appl. Phys. Lett. 92, 023514 (2008).

${ }^{19}$ A. Lupaşcu, E. F. C. Driessen, L. Roschier, C. J. P. M. Harmans, and J. E. Mooij, Phys. Rev. Lett. 96, 127003 (2006).

${ }^{20}$ I. Siddiqi, R. Vijay, M. Metcalfe, E. Boaknin, L. Frunzio, R. J. Schoelkopf, and M. H. Devoret, Phys. Rev. B 73, 054510 (2006).

${ }^{21}$ F. Mallet, F. Ong, A. Palacios-Laloy, F. Nguyen, P. Bertet, D. Vion, and D. Esteve, Nat. Phys. 5, 791 (2009).

${ }^{22}$ E. del Barco, A. D. Kent, E. C. Yang, and D. N. Hendrickson, Phys. Rev. Lett. 93, 157202 (2004).

${ }^{23}$ M. Jamet, V. Dupuis, P. Mélinon, G. Guiraud, A. Pérez, W. Wernsdorfer, A. Traverse, and B. Baguenard, Phys. Rev. B 62, 493 (2000). 
${ }^{24}$ M. I. Dykman and M. A. Krivoglaz, Physica A 104, 480 (1980).

${ }^{25}$ M. J. Feldman, P. T. Parrish, and R. Y. Chiao, J. Appl. Phys. 46, 4031 (1975).

${ }^{26}$ B. Yurke, L. R. Corruccini, P. G. Kaminsky, L. W. Rupp, A. D. Smith, A. H. Silver, R. W. Simon, and E. A. Whittaker, Phys. Rev. A 39, 2519 (1989).

${ }^{27}$ R. Vijay, Ph.D. thesis, Yale University, New Haven, CT (2008).

${ }^{28}$ M. Castellanos-Beltran, K. D. Irwin, G. C. Hilton, L. R. Vale, and K. W. Lehnert, Nat. Phys. 4, 929 (2008).

${ }^{29}$ N. Bergeal, F. Schackert, M. Metcalfe, R. Vijay, V. Manucharyan, L. Frunzio, D. Prober, R. J. Schoelkopf, S. Girvin, and M. Devoret, Nature (London) 465, 64 (2010).

${ }^{30}$ C. M. Caves, Phys. Rev. D 26, 1817 (1982).
${ }^{31}$ V. E. Manucharyan, E. Boaknin, M. Metcalfe, R. Vijay, I. Siddiqi, and M. Devoret, Phys. Rev. B 76, 014524 (2007).

${ }^{32}$ C. Laflamme and A. A. Clerk, Phys. Rev. A 83, 033803 (2011).

${ }^{33}$ P. H. Bryant, R. Movshovich, and B. Yurke, Phys. Rev. Lett. 66, 2641 (1991).

${ }^{34}$ F. Wellstood, C. Urbina, and J. Clarke, Appl. Phys. Lett. 50, 772 (1987).

${ }^{35}$ R. Vijay, J. D. Sau, M. L. Cohen, and I. Siddiqi, Phys. Rev. Lett. 103, 087003 (2009).

${ }^{36}$ R. Vijay, E. M. Levenson-Falk, D. H. Slichter, and I. Siddiqi, Appl. Phys. Lett. 96, 223112 (2010).

${ }^{37}$ B. Yurke, in Quantum Squeezing, edited by P. D. Drummond and Z. Ficek (Springer, New York, 2004), Chap. 3, p. 53. 